\title{
Solar flares: observations vs simulations
}

\author{
Fatima Rubio da Costa, ${ }^{1}$ Francesca Zuccarello, ${ }^{1}$ Nicolas Labrosse,${ }^{2}$ \\ Lyndsay Fletcher, ${ }^{2}$ Tomáš Prosecký ${ }^{3}$ and Jana Kašparová ${ }^{3}$ \\ ${ }^{1}$ Dipartimento di Fisica e Astronomia, Via S. Sofia, 78. 95123. Catania, Italy \\ ${ }^{2}$ University of Glasgow, School of Physics and Astronomy \\ Kelvin Building, University of Glasgow. G12 8QQ, Glasgow, UK \\ ${ }^{3}$ Astronomical Institute of the Academy of Sciences of the Czech Republic, v. v. i. \\ Fričova 298, 25165, Ondřejov, Czech Republic \\ email: frdc@oact.inaf.it
}

\begin{abstract}
In order to study the properties of faint, moderate and bright flares, we simulate the conditions of the solar atmosphere using a radiative hydrodynamic model (Abbett \& Hawley, 1999). A constant beam of non-thermal electrons is injected at the apex of a $1 \mathrm{D}$ coronal loop and heating from thermal soft X-ray emission is included. We compare the results with some observational data in Ly- $\alpha$ (using TRACE 1216 and $1600 \AA$ data and estimating the "pure" Ly- $\alpha$ emission) and in $\mathrm{H} \alpha$ (data taken with a Multichannel Flare Spectrograph, at the Ondrejov Observatory).
\end{abstract}

Keywords. Methods: numerical, radiative transfer, Sun: atmosphere, Sun: flares

\section{Introduction}

The NLTE Radyn code models a 1D plane-parallel atmosphere using the plane-parallel equation of radiative hydrodynamics (Carlsson \& Stein, 1997).

We obtain the non-LTE solution of the population equations for hydrogen, helium, single ionized calcium and single ionized magnesium atoms, assuming complete redistribution. We calculate the evolution of the atmospheric plasma parameters, and selected radiated line intensities as a function of time and we compare the results obtained for the hydrogen atom with the intensity measured in $\mathrm{Ly}-\alpha$ and $\mathrm{H} \alpha$ during a solar flare to test agreement between observations and the simulations.

\section{Simulations}

A constant beam of non-thermal electrons is injected at the apex of a $1 \mathrm{D}$ coronal loop and heating from thermal soft X-ray and UV emission is included (Abbett \& Hawley, 1999). Cooling resulting from bremsstrahlung and collisionally excited metal transitions is also added. The non-thermal electron beam is assumed to have a power-law distribution with a spectral index $\delta=4$ and a low-energy cutoff of $E_{c}=20 \mathrm{keV}$.

To investigate the properties of the lower atmosphere during the impulsive phase of a solar flare, the code takes into account three levels of non-thermal electron energy flux:

- $\mathcal{F}=10^{9}$ ergs $\mathrm{cm}^{-2} \mathrm{~s}^{-1}$, hereafter called run F9, which corresponds to weak flares. The quiescent atmosphere is heated for $67.1 \mathrm{~s}$.

- $\mathcal{F}=10^{10} \mathrm{ergs} \mathrm{cm}^{-2} \mathrm{~s}^{-1}$, run F10, which simulates moderate flares, for $45.2 \mathrm{~s}$.

- $\mathcal{F}=10^{11}$ ergs $\mathrm{cm}^{-2} \mathrm{~s}^{-1}$, run F11, associated to strong flares. This case is computationally much more problematic and the run is only for $1.62 \mathrm{~s}$.

Fig. 1 shows the evolution of the temperature stratification for the three different flare cases; the dotted line is the temperature of the initial preflare atmosphere $($ at $t=0$ 

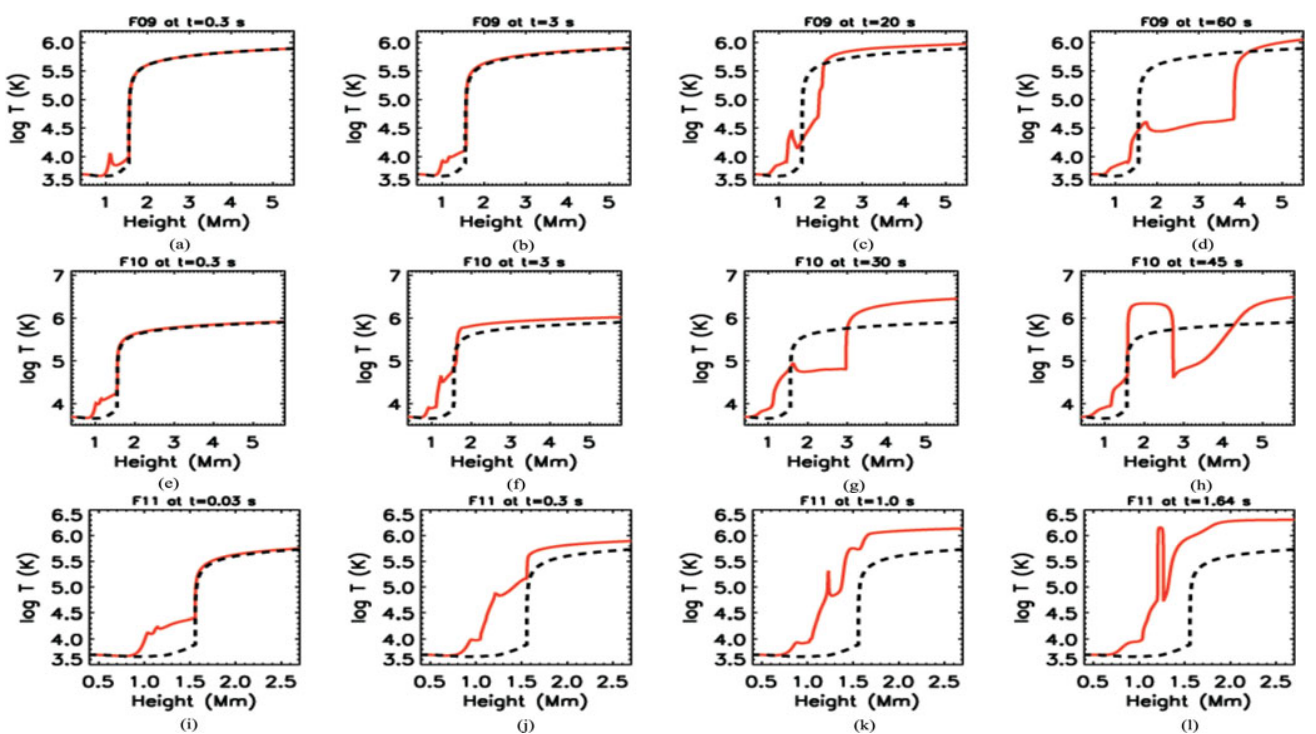

Figure 1. Evolution of the temperature stratification during the F09 (upper pannel), F10 (middle pannel) and F11 (bottom pannel) runs. The dotted line represents the temperature of the initial preflare atmosphere and the solid line reports the temperature during the flare.

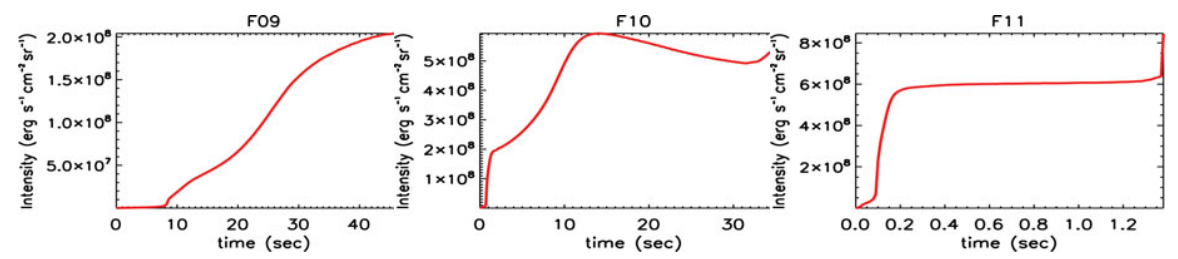

Figure 2. Ly- $\alpha$ integrated intensity as a function of time for the three different flare models.

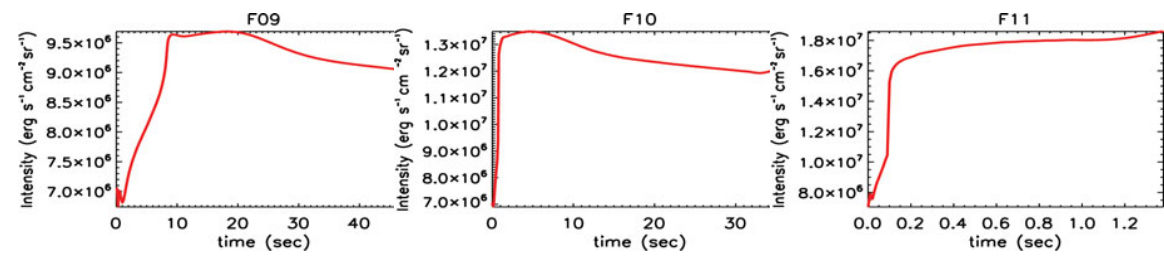

Figure 3. $\mathrm{H} \alpha$ integrated intensity as a function of time for the three different flare models.

seconds) and the solid line is the temperature stratification at each time during the flare. Moderate and Strong flares have an initial gentle phase (see panels (f) and (j)) and a subsequent explosive phase (see panels (h) and (l)), characterized by large material flows, while Weak flares do not have enough energy to produce the explosive phase.

We calculate the radiative emission in $\mathrm{H} \alpha$ and $\mathrm{Ly}-\alpha$ from the chromospheric footpoints of the flare. The line intensity is obtained by integrating the line profiles over an interval of $\Delta \lambda=5 \AA$. The intensity of the non-thermal electron beam affects the Ly- $\alpha$ intensity in time (Fig. 2), being only a factor 4 higher for F11 than for F09. Fig. 3 shows the $\mathrm{H} \alpha$ light curve during the flare for each run. The $\mathrm{H} \alpha$ intensity varies by less than a factor of 2 between F09 and F11. 


\begin{tabular}{c|cc} 
& M6.6 Flare & M1.4 Flare \\
\hline$I_{L_{\alpha}}\left(\mathrm{erg} \mathrm{s}^{-1} \mathrm{~cm}^{-2} \mathrm{sr}^{-1}\right)$ & $9.9 \times 10^{5}$ & $1.4 \times 10^{7}$ \\
\hline
\end{tabular}

Table 1. Intensity estimated at the beginning of the impulsive phase at the flare footpoints for two flares observed by TRACE.

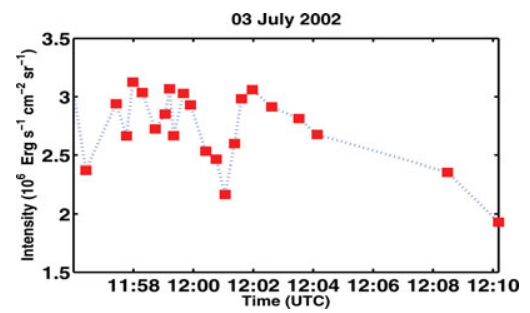

Figure 4. $\mathrm{H} \alpha$ light curve obtained integrating the intensity along the line profile $(\Delta \lambda=5 \AA)$.

\section{Observations}

We studied two different flares using TRACE data: an M1.4 class flare that occurred on 08 September 1999 at 12:08 UT and an M6.6 class flare that occurred on 28 February 1999 at 16:31 UT. A cleaned version of the $\mathrm{Ly}-\alpha$ image is obtained by correcting the influence of the $1600 \AA$ TRACE channel on the $1216 \AA$ TRACE channel using: $I_{L y_{\alpha}}=$ $0.97 \times I_{1216}-0.14 \times I_{1600}(\mathrm{Kim}$ et al., 2006). Measuring the count rate in a region of $250 \times 250$ pixels $^{2}$ at the footpoints of the flare in the corrected Ly- $\alpha$ image (using a threshold range between 1200 and $4090 \mathrm{DN}$ ), we estimate the intensity in the flare in Ly- $\alpha$ (see Table 1) (Rubio da Costa et al., 2009).

A C.3 class flare that occurred on 03 July 2002 at 11:43 UT was observed at the Ondrejov Observatory, with the Multichannel Flare Spectrograph (MFS), from 11:56:24 UT to $12: 10: 42$ UT. We calibrated the data obtaining the intensity at different wavelengths along the $\mathrm{H} \alpha$ line profile at different times, determining therefore the evolution of the $\mathrm{H} \alpha$ line profile during the flare for $\Delta \lambda=5 \AA$ (see Fig. 4). This can be directly compared to calculated values.

\section{Conclusions}

The Ly- $\alpha$ intensity estimated from TRACE images is comparable with the theoretical one obtained from the RADYN code even if during the simulations the energy flux of the non-thermal electron beam is constant in time.

The values of the $\mathrm{H} \alpha$ light curve obtained from observations are similar to the simulated ones. The behaviour of the two light curves is different (compare Fig. 3 with Fig. 4): this might be explained by the time variation of the beam flux.

\section{Acknowledgements}

Financial support by the European Commission through the SOLAIRE Network (MTRNCT-2006-035484) is gratefully acknowledged.

\section{References}

Abbett, W. P. \& Hawley, S. L. 1999, ApJ, 521, 906

Carlsson, M. \& Stein, R. F. 1997, ApJ, 481, 500

Kim, S. S., Roh, H.-S., Cho, K.-S., \& Shin, J. 2006, A\&A, 456, 747

Rubio da Costa, F., Fletcher, L., Labrosse, N., \& Zuccarello, F. 2009, A\&̛A, 507, 1005 